\title{
Polymer-based Hydrophobic Nanostructures for Hydrogen Gas Sensing Application
}

\author{
Mohammad K. Hossain ${ }^{1}$, Qasem A. Drmosh ${ }^{2}$ \\ ${ }^{1}$ Center of Research Excellence in Renewable Energy (CoRERE), Research Institute, King Fahd \\ University of Petroleum \& Minerals, (KFUPM), Dhahran 31261, Saudi Arabia, \\ ${ }^{2}$ Center of Research Excellence in Nanotechnology (CENT), Research Institute, King Fahd University \\ of Petroleum \& Minerals, (KFUPM), Dhahran 31261, Saudi Arabia \\ kamalhossain@kfupm.edu.sa, drmosh@kfupm.edu.sa
}

\begin{abstract}
:
A simple and hand-on two-step process has been implemented to fabricate polymer-based hydrophobic nanostructures for hydrogen gas sensing application. Topographic measurements confirmed irregular hills and dips of various dimensions that play a vital role to create air-bubble pockets yielding a hydrophobic surface atop. Wetting contact angle (WTC) measurements further revealed the contact angle to be $130.0^{\circ} \pm 10.0^{\circ}$. Such nanostructures were expected to provide a template for gas sensing materials of higher surface area that is very essential to devise an efficient and sensitive gas sensing platform at room temperature. From this direction, a very thin layer pf palladium, ca. $40 \mathrm{~nm}$ of thickness was sputtered. Thereafter, further topographic and WTC measurements were carried out. It was observed that the nanostructures and contact angles remain almost same. Higher sensitivity to $\mathrm{H}_{2}$ gas sensing was observed. The surface nanostructures were found very stable and durable over the time of six months and beyond. A polymer-based hydrophobic gas sensing platform as investigated in this study will play dual role of hydrophobicity as well as superior gas sensing characteristics.
\end{abstract}

Key words: hydrogen, superhydrophobic, sensing, nanostructures, palladium.

\section{Introduction}

Hydrogen $\left(\mathrm{H}_{2}\right)$ has been an important energy carrier that is going to be complementary to current electricity very soon [1]. A persistence challenge is being carried out to incorporate $\mathrm{H}_{2}$ as a fuel for 'zero-emissions' vehicles, to heat accommodations and workplaces, to fuel aircraft, etc. [1-2]. Therefore, an efficient and highly sensitive $\mathrm{H}_{2}$ is in urgent need for safe deployment of all $\mathrm{H}_{2}$-based applications. Most of $\mathrm{H}_{2}$ gas sensor, particularly used in industries and workplaces are not suitable for advanced and sophisticated applications. The sensor needs to be smart, compact and durable along with ultrashort response time. Amongst many gas sensing materials, palladium $(\mathrm{Pd})$ is the perfect one for $\mathrm{H}_{2}$ gas sensing because of its higher selectivity and sensitivity to $\mathrm{H}_{2}$ [3-4].

Here in this study, we have adopted a simple and inexpensive two-step strategy to fabricated polymer-based hydrophobic nanostructures followed by sputtering of $\mathrm{Pd}$ thin film atop. Wetting contact angle measurements confirmed higher contact angle as well as sensitivity to $\mathrm{H}_{2}$ gas was carried out in a customized setup.

\section{Methods and Materials}

Commercially available polycarbonate sheet was treated with diluted acetone under controlled lab conditions. Topography of asfabricated nanostructures obtained thereafter was characterized by scanning electron microscope (SEM) and Dektak profilometer. Ultrathin layer of $\mathrm{Pd}$ was deposited by $\mathrm{DC}$ sputtering system (NSC4000-Nanomaster) with the chamber background pressure as low as $3.5 \times 10^{-6}$ Torr. The deposition power was maintained to be $30 \mathrm{~W}$ for $20 \mathrm{~s}$. A customized gas chamber, Linkam stage (Model HFS-600EPB4, UK) was used to incorporate air and hydrogen balanced nitrogen $\left(1 \% \mathrm{H}_{2}, 99 \% \mathrm{~N}_{2}\right)$ sequentially at room temperature. Two mass flow controllers (MFCs) connected with an external X PH-100 power hub supply were utilized to control the flow of $\mathrm{H}_{2}$. The sensing measurements were performed at room temperature. 

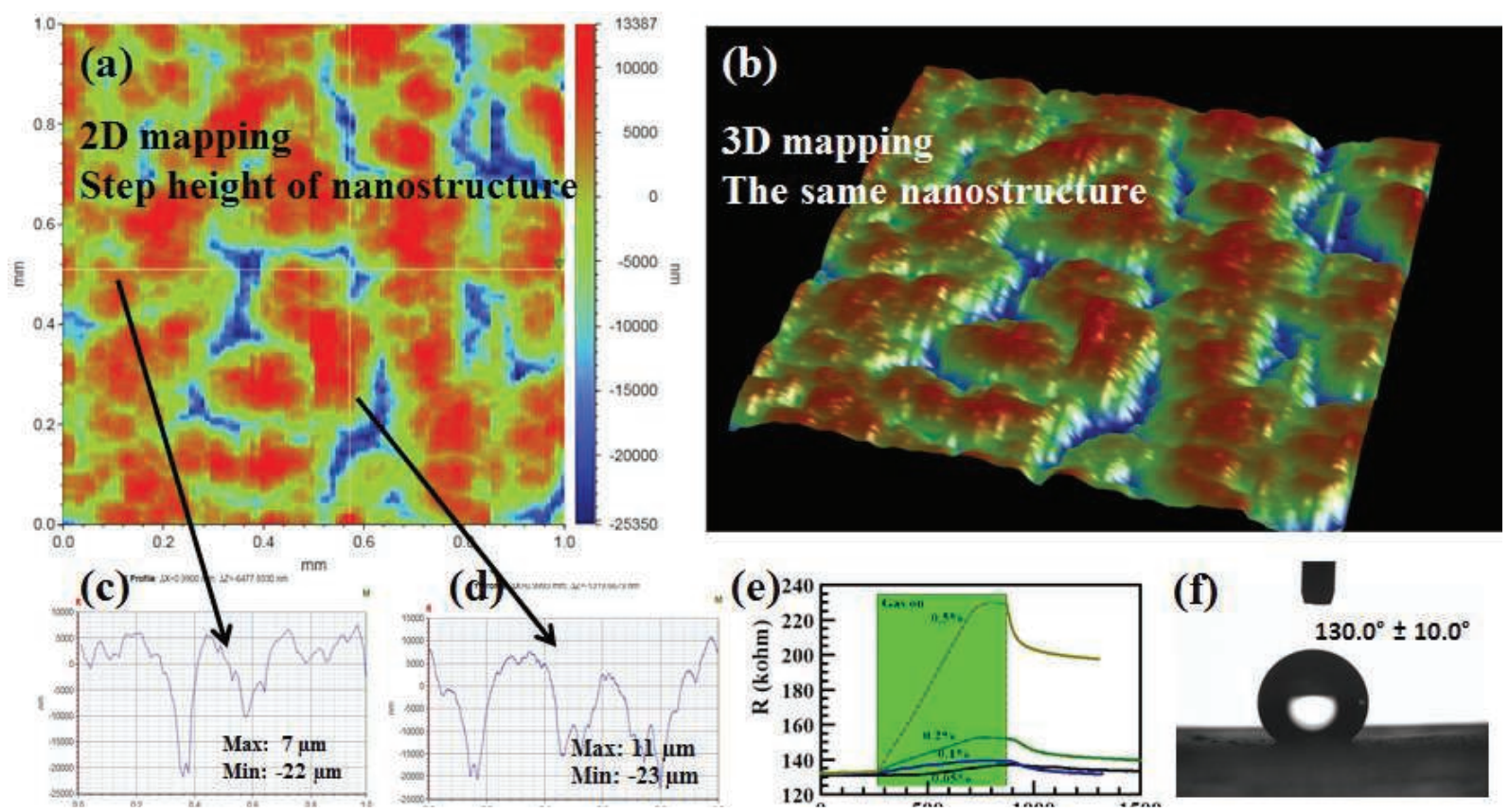

Fig. 1 (a) 2D mapping of hte treated polymer, (b) 3D hawk-eye view showing hills and dips, (c)-(d) line profiles along $\mathrm{x}$-axis and $\mathrm{y}$-axis as shown in Fig. $1 \mathrm{a},\left((\mathrm{e})\right.$ Response to $\mathrm{H}_{2}$ gas sensing, and (f) contact angle of asfabricated poymer nanostructures.

\section{Results and discussion}

A typical line profiles along two axes was shown in Fig. 1c and Fig. 1d. WTC measurements were carried out before and after the $\mathrm{Pd}$ deposition on polymer-based nanostructures. The measurements revealed that the contact angle remain almost same as mentioned in Fig, 1f. A prompt response to $\mathrm{H}_{2}$ gas sensing was observed as shown in Fig. $1 \mathrm{e}$. As can be seen, the resistance of the Pd film increased with exposing $\mathrm{H}_{2}$ into the test chamber and reached to a maximum value and decreased down while purging $\mathrm{N}_{2}$ into the measurement cell. Further characterizations through detailed SEM observations along with UV-vis spectroscopy and gas sensing characteristics are out of this context because of space constrain. Details will be presentenced in the event.

\section{Conclusion}

Polymer-based hydrophobic nanostructures have been fabricated for hydrogen gas sensing application. Wetting contact angle (WTC) measurements revealed the contact angle to be $130.0^{\circ} \pm 10.0^{\circ}$. Detailed dimension of air-bubble pockets as observed herewith were sufficient to provide moderate to high contact angle. A very thin layer of Pd, ca. $100 \mathrm{~nm}$ of thickness was sputtered atop. WTC measurements before and after the deposition confirmed that contact angle remained almost same. Sensing characteristics to $\mathrm{H}_{2}$ gas sensing as carried out in a customized setup revealed a prompt response to the target gas. The surface nanostructures were found very stable and durable over the time of six months and beyond. Such a polymer-based hydrophobic gas sensing platform as investigated in this study will play dual role of hydrophobicity as well as gas sensing in application specific needs.

\section{Acknowledgement}

Author acknowledges Center of Research Excellence in Renewable Energy (CoRERE), Research Institute, King Fahd University of Petroleum \& Minerals, (KFUPM), Dhahran 31261, Saudi Arabia. QAD likes to acknowledge Center of Research Excellence in Nanotechnology (CENT).

\section{References}

[1] F.J. Vivas, A. De las Heras, F. Segura, J.M. Andújar, A review of energy management strategies for renewable hybrid energy systems with hydrogen backup, Renewable and Sustainable Energy Reviews 82, 126-155 (2018); doi: 10.1016/j.rser.2017.09.014.

[2] C. B. Robledo, V. Oldenbroek, F. Abbruzzese, A. J.M. van Wijk, Integrating a hydrogen fuel cell electric vehicle with vehicle-to-grid technology, photovoltaic power and a residential building, Applied Energy 215, 615-629(2018); doi: 10.1016/j.apenergy.2018.02.038.

[3] M. Fisser, R. A. Badcock, P. D. Teal, A, Hunzea, Optimizing the sensitivity of palladium based hydrogen sensors, Sensors and Actuators B 259, 10-19 (2018); doi: /10.1016/j.snb.2017.11.180.

[4] J. Noh, J. M. Lee, W. Lee, Low-Dimensional Palladium Nanostructures for Fast and Reliable Hydrogen Gas Detection, Sensors 11, 825-851 (2011); doi:10.3390/s110100825. 